\title{
Least Order Coprime Factorizations of Rational Matrices: The Canonical Case
}

\author{
Cristian OARĂ ${ }^{1}$ \\ University Polytechnica Bucharest \\ Faculty of Automatic Control and Computers \\ Austrului 34, RO-73115, \\ Bucharest, Romania \\ cris@indinf .pub.ro
}

\author{
Andras VARGA \\ German Aerospace Center \\ DLR - Oberpfaffenhofen \\ Institute of Robotics and System Dynamics \\ D-82234 Wessling, Germany \\ Andras.Varga@dlr.de
}

\begin{abstract}
For a given rational matrix $G$ with complex coefficients and a given domain $\Gamma$ in the closed complex plane, both arbitrary, we develop a complete theory of coprime factorizations of $G$ over $\Gamma$, with denominators of McMillan degree as small as possible. We consider both the cases in which the denominator is arbitrary and in which it has a certain symmetry, namely it is $J$ all-pass, either with respect to the imaginary axis or to the unit circle. All the developments are carried out in terms of descriptor realizations associated with rational matrices, leading to explicit and computationally efficient formulas.
\end{abstract}

\section{Introduction}

Let $G$ be an arbitrary rational matrix (possible improper) and let $\Gamma$ be a given domain of the closed complex plane. A left coprime factorization (LCF) over $\Gamma$ of $G$ is a fractional representation of the form $G=M^{-1} N$, with $N$ and $M$ rational matrices having poles only in $\Gamma$ and satisfying $M U+N V=I$ for certain rational matrices $U$ and $V$ with all poles in $\Gamma$. Analogously, a factorization in the form $G=N M^{-1}$, with $N$ and $M$ having poles only in $\Gamma$, and satisfying $U M+V M=I$ for certain rational matrices $U$ and $V$ with poles in $\Gamma$ is called a right coprime factorization.

In this paper we develop a complete theory of LCFs over $\Gamma$ with denominators $M$ of smallest possible McMillan degree (see the precise definition below). For brevity, we call such factorizations of least order, and define the order (or the degree) of the factorization to be the McMillan degree of the denominator $M$. We address only LCFs since all results for right coprime factorizations follow by duality.

\footnotetext{
${ }^{1}$ Corresponding author. The work of Cristian Oară was completed with the support of Alexander von Humboldt Foundation, Bonn, Germany
}

Apparently, the theory of least order coprime factorizations has not been previously considered as such in the literature, although it is crux to solving various problems encountered in the theory of linear systems and networks [14], to canonical and noncanonical spectral factorizations of unstable rational matrices [2], to several conjugation based approaches to nonstandard $H_{\infty}$ control problems $[15,16]$, and brings important numerical advantages as for instance in computing coprime factorizations with denominators which are either polynomial, proper, stable, all-pass, or $J$ lossless. The only noticeable exception is [1], where coprime factorizations are addressed with another requirement of minimality, namely the sum of the McMillan degrees of the denominator and nominator to be as small as possible. However, even in the quite particular case treated in [1] where the matrix to be factored is square and of full rank over rationals this sum-minimality leads to combinatorial problems over an infinite set which have little interest from an algorithmic viewpoint as well as for the type of applications that we have mentioned above.

Our main tool in the derivations - which has also an independent interest - is a general pole displacement theorem which gives a characterization in terms of realizations for an invertible rational matrix $M$ to cancel in the product $M G$ all poles of $G$ in $\Gamma$. An important feature of our result is the possibility to cope with domains containing infinity, without using conformal mapping techniques. In particular, we show that the least order of a LCF over $\Gamma$ is $n_{b}$, where $n_{b}$ is the number of poles of $G$ outside $\Gamma$, and give a description in terms of realizations of all factors solving the least order LCF. It turns out that the basic ingredient in computing LCFs of least order lies in the solution of a generalized eigenvalue assignment problem of order $n_{b}$.

We develop also a theory of least order LCFs with the additional requirement that the denominator has a certain symmetry. We consider here two cases, in which the denominator is $J$ all-pass, either with respect to the imaginary axis or to the unit circle, and the domain $\Gamma$ 
is adequately chosen to reflect the respective symmetry. We study here only LCFs with $J$ all-pass denominator of degree $n_{b}$ which we call the canonical case, and give necessary and sufficient solvability conditions. When solutions exist we give a parametrized description of the factors in terms of associated realizations. When such canonical solutions do not exist, an interesting question is to study the solution of noncanonical LCFs with $J$ all-pass denominators of McMillan degree as small as possible $\left(>n_{b}\right)$. Due to lack of space we treat in this paper only the canonical case, while the noncanonical case will be reported in a future work. The computation of factors in the canonical case relies on solving a generalized Lyapunov equation of order $n_{b}$. The canonical case treated here has important connections with the canonical Wiener-Hopf and $J$ spectral factorizations as discussed in [2] for proper and invertible rational matrices. Another connected work is [16] which in contrast to our results is restricted to the case with $J$ lossless denominator with respect to the imaginary axis, and involves the solution of a Riccati equation of order equal to the McMillan degree of $G$.

\section{Preliminaries}

By $\mathbb{C}, \mathbb{C}^{-}, \mathbb{C}^{+}, \mathbb{C}^{0}$, and $\mathbb{R}$ we denote the complex plane, the open left half plane, the open right half plane, the imaginary axis, and the real axis, respectively, and let $\overline{\mathbb{C}}:=\mathbb{C} \cup\{\infty\}$ be the closed complex plane, and $\overline{\mathbb{C}}^{-}:=\mathbb{C}^{-} \cup \mathbb{C}^{0} \cup\{\infty\}, \overline{\mathbb{C}}^{+}:=$ $\mathbb{C}^{+} \cup \mathbb{C}^{0} \cup\{\infty\}, \overline{\mathbb{C}}^{0}:=\mathbb{C}^{0} \cup\{\underline{\infty}\}$. By $\mathbb{D}$ we denote the open unit disk and $\mathbb{D}^{c}=\overline{\mathbb{C}} \backslash \overline{\mathbb{D}}$ stands for the exterior of the closed unit disk, containing the infinity.

Consider the disjoint partition of the closed complex plane into a "good" region $\Gamma_{g}$ and a "bad" region $\Gamma_{b}$ as

$$
\overline{\mathbb{C}}=\Gamma_{g} \cup \Gamma_{b}
$$

A frequent interpretation of $\Gamma_{g}$ in system theory is related to the standard stability concept, that is, for linear continuous-time systems $\Gamma_{g}=\mathbb{C}^{-}$, while for linear discrete-time systems $\Gamma_{g}=\mathbb{D}$ (or, sometimes, their closures). However, we use also other interpretations, as for instance $\Gamma_{g}=\{\infty\}$ to obtain coprime factorizations with polynomial factors. When dealing with LCFs of rational matrices with real coefficients, we assume that $\Gamma_{g}$ is symmetric with respect to the imaginary axis, that is, if $\lambda \in \Gamma_{g}$ then $\bar{\lambda} \in \Gamma_{g}$. This is in particular true for all the aforementioned examples of $\Gamma_{g}$ and guarantees that the resulting factors of the LCF have real coefficients as well.

Throughout the paper we consider rational matrices with coefficients in the field $\mathbb{F}$, where $\mathbb{F}$ denotes either $\mathbb{R}$ or $\mathbb{C}$. We denote with $G^{\sim}$ the adjoint of $G$, where $G^{\sim}(s)=G^{*}(-\bar{s})$ in continuous-time and
$G^{\sim}(z)=G^{*}(1 / \bar{z})$ in discrete-time. In particular, if $G$ has real coefficients we have $G^{\sim}(s)=G^{T}(-s)$ and $G^{\sim}(z)=G^{T}(1 / z)$, respectively. Let $J$ be a signature matrix, i.e., a matrix satisfying $J=J^{*}=J^{-1}$. We say that the rational matrix $G$ is $J$ all-pass with respect to the imaginary axis if $G^{\sim} J G=J$, where $G^{\sim}$ denotes the adjoint in continuous-time. Accordingly, we say that $G$ is $J$ all-pass with respect to the unit circle if $G^{\sim} J G=J$, where $G^{\sim}$ denotes now the adjoint in discrete-time. By definition, the McMillan degree of $G$-denoted $\delta(G)$ - is the sum of the orders of all its poles (finite and infinite). Once a partition (1) is fixed, we have $\delta(G)=n_{b}+n_{g}$, where $n_{g}$ denotes the number of "good" poles in $\Gamma_{g}$ and $n_{b}$ denotes the number of "bad" poles in $\Gamma_{b}$ (counting multiplicities).

It is well known (see for example $[13,12]$ ) that any $p \times m$ rational matrix $G(\lambda)$ with coefficients in $\mathbb{F}$ (even improper or polynomial) has a descriptor realization of the form

$$
G(\lambda)=D+C(\lambda E-A)^{-1} B=:\left[\begin{array}{c|c}
A-\lambda E & B \\
\hline C & D
\end{array}\right]
$$

where $A, E \in \mathbb{F}^{n \times n}, B \in \mathbb{F}^{n \times m}, C \in \mathbb{F}^{p \times n}, D \in$ $\mathbb{F}^{p \times m}$, and the so called pole pencil $A-\lambda E$ is regular, i.e., it is square and $\operatorname{det}(A-\lambda E) \not \equiv 0$. The dimension $n$ of the square matrices $A$ and $E$ is called the order of the realization (2). We use $\Lambda(A-\lambda E)$ to denote the union of generalized eigenvalues of the regular pencil $A-\lambda E$ (finite and infinite, multiplicities counting). The descriptor realization (2) of $G$ is called minimal if its order is as small as possible among all realizations of this kind. For a minimal descriptor realization (2) of order $n$ we have $\delta(G)=\operatorname{rank} E \leq n$.

The principal inconvenience of realizations of the form (2) is that their minimal possible order is greater than the McMillan degree of $G$, unless $G$ is proper, and this brings important technical difficulties in factorization problems in which the McMillan degree plays a paramount role. A remedy to this is to use a generalization of (2) in which either the " $B$ " or the " $C$ " matrix is replaced by a matrix pencil, as explained furher (see [6] for a detailed discussion).

Any rational $p \times m$ matrix $G$ has a realization

$$
G(\lambda)=D+C(\lambda E-A)^{-1}(B-\lambda F)=:\left[\begin{array}{c|c}
A-\lambda E B-\lambda F \\
\hline C & D
\end{array}\right]
$$

and for any fixed $\alpha, \beta \in \mathbb{F}$, not both zero, there exists a realization

$G(\lambda)=D+C(\lambda E-A)^{-1} B(\alpha-\lambda \beta)=:\left[\begin{array}{c|c}A-\lambda E B(\alpha-\lambda \beta) \\ \hline C & D\end{array}\right]$

where $A, E \in \mathbb{F}^{n \times n}, B, F \in \mathbb{F}^{n \times m}, C \in \mathbb{F}^{p \times n}$, $D \in \mathbb{F}^{p \times m}$, and the pole pencil $A-\lambda E$ is regular. A realization (4) will be called centered at $\frac{\alpha}{\beta}$ (if $\beta=0$ 
we interpret $\frac{\alpha}{\beta}$ as $\infty$ ). We call realizations of the type (3) or (4) minimal if the dimension of the square matrices $A$ and $E$ (also called the order of the realization) is as small as possible among all realizations of the respective kind. It can be easily shown that any rational matrix $G(\lambda)$ has a minimal realization of type (3) of order equal to $\delta(G)$. For any fixed $\alpha$ and $\beta$, not both zero, and such that $\frac{\alpha}{\beta}$ is not a pole of $G$ there also exists a minimal realization of type (4) of order equal to $\delta(G)$. For the rest of the paper, if not otherwise stated, we assume this choice implicitly. The nice feature of (3) and (4) that their minimal order equals the McMillan degree of $G$ recommends them for the kind of problems treated in this paper. For a detailed discussion of such "nonstandard" descriptor realizations we refer to [6]. The following result (well-known for standard descriptor realizations [7], [13]) will be instrumental in the sequel.

Theorem 2.1 Let $G(\lambda)$ be an arbitrary rational matrix with a realization (3) (or (4))). Then the poles of $G(\lambda)$ are among the generalized eigenvalues of the pole pencil $A-\lambda E$. If the realization (3) is minimal (or if $\frac{\alpha}{\beta}$ is not a pole of $G(\lambda)$, and (4) is minimal) then the poles of $G(\lambda)$ are exactly the generalized eigenvalues of $A-\lambda E$, and the orders of the poles are pairwise equal to the multiplicities of the generalized eigenvalues of $A-\lambda E$.

Once a partition (1) is fixed, we assume that $G$ is given by a separated realization with respect to (1), namely

$$
G(\lambda)=\left[\begin{array}{cc|c}
A_{b}-\lambda E_{b} & A_{b g}-\lambda E_{b g} & B_{b} \\
O & A_{g}-\lambda E_{g} & B_{g} \\
\hline C_{b} & C_{g} & D
\end{array}\right]
$$

where the $n_{b} \times n_{b}$ pencil $A_{b}-\lambda E_{b}$ contains the $n_{b}$ poles of $G(\lambda)$ in $\Gamma_{b}$ and

$$
\operatorname{rank}\left[\begin{array}{ll}
E_{b} & E_{b g}
\end{array}\right]=n_{b},
$$

that is, all infinite nondynamic modes are included in $A_{g}-\lambda E_{g}$. Starting with an arbitrary minimal realization (2) it is always possible to arrive to a separated realization by determining first a realization that separates the infinite nondynamic modes and further making a spectral decomposition of the pole pencil with respect to the partition (1). Furthermore, if the realization to start with has real coefficients, and $\Gamma_{g}$ is symmetric, we can always determine a separated realization with real coefficients.

\section{Basic Pole Displacement Result}

In this section we solve the following general pole displacement problem (PDP).
PDP: Given a $p \times m$ rational matrix $G$ and a disjoint partition $\overline{\mathbb{C}}=\Gamma_{g} \cup \Gamma_{b}$, both arbitrary, determine an invertible $p \times p$ rational matrix $M$ with all poles in $\Gamma_{g}$ which cancels by left multiplication all poles of $G$ located in $\Gamma_{b}$, i.e., such that $N(\lambda):=M(\lambda) G(\lambda)$ has all poles in $\Gamma_{g}$.

The following theorem gives an answer to the PDP in terms of descriptor realizations of $G$ and $M$. It is a rich extension of Theorem 3.1 and Lemma 4.1 in [9].

Theorem 3.1 Given a rational matrix $G(\lambda)$ and a disjoint partition $\overline{\mathbb{C}}=\Gamma_{g} \cup \Gamma_{b}$, both arbitrary, let $n_{b}$ be the number of poles of $G$ in $\Gamma_{b}$. Assume (5) is a minimal descriptor realization of $G$ separated with respect to the given partition and satisfying the condition (6). Then the class of solutions to the PDP is given by

$$
M(\lambda)=\left[\begin{array}{c|c}
A_{x}-\lambda E_{x} & B_{x}(\alpha-\beta \lambda) \\
\hline C_{x} & D_{x}
\end{array}\right]
$$

where the realization (7) is minimal and satisfies: $A_{x}-$ $\lambda E_{x}$ has all eigenvalues in $\Gamma_{g}, D_{x}$ is invertible, $\frac{\alpha}{\beta} \notin$ $\Lambda\left(A_{b}-\lambda E_{b}\right)$, and

$$
\left[\begin{array}{cc}
A_{x}-\lambda E_{x} & B_{x}(\alpha-\beta \lambda) \\
C_{x} & D_{x}
\end{array}\right]\left[\begin{array}{c}
X \\
C_{b}
\end{array}\right]=\left[\begin{array}{c}
Y \\
O
\end{array}\right]\left(A_{b}-\lambda E_{b}\right)
$$

holds for certain injective matrices $X$ and $Y$. The minimal McMillan degree of a solution to the PDP is $n_{b}$.

\section{Least Order Degree Coprime Factorization}

For a given rational matrix $G(\lambda)$ and a domain $\Gamma_{g}$, both arbitrary, we characterize now the class of LCFs over $\Gamma_{g}$ having least order which we show to be $n_{b}$. For our proofs, we need the following result on the solution in a particular form to the generalized eigenvalue assignment problem.

Lemma 4.1 Let $A-\lambda E$ be a regular pencil, with $A$, $E \in \mathbb{F}^{n \times n}, B \in \mathbb{F}^{n \times m}$, let $\Gamma \subset \overline{\mathbb{C}}$ be a set of $n$ elements (not necessarily distinct, and assumed to be symmetric if $\mathbb{F}=\mathbb{R}$ ), and let $\alpha, \beta \in \mathbb{F}$, not both zero, such that $\frac{\alpha}{\beta} \notin \Lambda(A-\lambda E)$ and $\frac{\alpha}{\beta} \notin \Gamma$. Assume that:

(i) $\operatorname{rank}\left[\begin{array}{ll}A-\lambda E & B\end{array}\right]=n, \forall \lambda \in \mathbb{C} ;$ (ii) $\operatorname{rank}\left[\begin{array}{ll}E & B\end{array}\right]=n$. Then there exists a matrix $F \in$ $\mathbb{F}^{m \times n}$ such that $\Lambda(A-\lambda E+B F(\alpha-\beta \lambda))=\Gamma$.

Theorem 4.2 Given a rational matrix $G(\lambda)$ and a disjoint partition $\overline{\mathbb{C}}=\Gamma_{g} \cup \Gamma_{b}$, both arbitrary, let $n_{b}$ be the number of poles of $G$ in $\Gamma_{b}$. Assume (5) is a minimal descriptor realization of $G$ separated with respect to the given partition and satisfying the condition (6). Then: 1. The least order of a LCF of $G$ over $\Gamma_{g}$ is $n_{b}$. 
2. The class of solutions to the least order $L C F$ problem $G(\lambda)=M^{-1}(\lambda) N(\lambda)$ is given by

$$
M(\lambda)=\left[\begin{array}{c|c}
A_{b}-\lambda E_{b}+K C_{b}(\alpha-\lambda \beta) & K(\alpha-\lambda \beta) \\
\hline W C_{b} & W
\end{array},\right.
$$

and $N(\lambda)=M(\lambda) G(\lambda)$, where $W$ is an arbitrary invertible matrix, $K$ is any matrix that solves the generalized eigenvalue assignment problem $\Lambda\left(A_{b}-\lambda E_{b}+\right.$ $\left.K C_{b}(\alpha-\lambda \beta)\right) \subset \Gamma_{g}$, and $\alpha$ and $\beta$ are chosen to satisfy the requirements of Lemma 4.1. If $G$ has real coefficients and $\Gamma_{g}$ is a symmetric set then the class of solutions with real coefficients to the least order LCF problem is also given by the above formulas where now all the intervening elements can be chosen real.

\section{LCF with $J$ all-pass denominator with respect to the imaginary axis}

In this section we solve the least order LCF with the additional requirement on the denominator to have a certain symmetry, namely we consider here the case in which the denominator is $J$ all-pass with respect to the imaginary axis. To reflect this symmetry accordingly, we take throughout this section the disjoint partition $\overline{\mathbb{C}}=\Gamma_{g} \cup \Gamma_{b}$ defined by

$$
\Gamma_{b}:=\mathbb{C}^{+}\left(\text {or } \Gamma_{b}:=\mathbb{C}^{-}\right) \quad \Gamma_{g}:=\overline{\mathbb{C}} \backslash \Gamma_{b} .
$$

However, due to the additional requirement on the denominator to be $J$ all-pass it is not always possible to solve the LCF over $\Gamma_{g}$ with least order $n_{b}$. When this is possible, we call the factorization canonical, otherwise we call it noncanonical.

The following result that will prove useful later gives a characterization of proper $J$ all-pass rational matrices in terms of minimal realizations.

Lemma 5.1 Let $M(s)$ be a proper square invertible rational matrix, having a minimal realization

$$
M(s):=\left[\begin{array}{c|c}
A-s E & B \\
\hline C & D
\end{array}\right]
$$

Then $M$ is $J$ all-pass with respect to the imaginary axis if and only if $D^{*} J D=J$ and there exists an invertible Hermitic matrix $X$ such that $A^{*} X E+E^{*} X A-C^{*} J C=$ 0 , and $C-D J B^{*} X E=0$.

The following theorem gives a complete solution to the LCF problem with $J$ all-pass denominator of McMillan degree $n_{b}$ (the canonical case).

Theorem 5.2 Given an arbitrary rational matrix $G(s)$ and a disjoint partition $\overline{\mathbb{C}}=\Gamma_{g} \cup \Gamma_{b}$ defined by
(10), let $n_{b}$ be the number of poles of $G$ in $\Gamma_{b}$. Assume (5) is a minimal descriptor realization of $G$ separated with respect to the given partition and satisfying the condition (6). Then the LCF with $J$ all-pass denominator with respect to the imaginary axis has a solution $G(s)=M^{-1}(s) N(s)$ of least order $n_{b}$ if and only if the equation

$$
A_{b}^{*} X E_{b}+E_{b}^{*} X A_{b}-C_{b}^{*} J C_{b}=0
$$

has an invertible Hermitic solution $X$. In this case, the class of all solutions is given by

$$
M(s)=\left[\begin{array}{c|c}
A_{b}+K C_{b}-s E_{b} & K \\
\hline W C_{b} & W
\end{array},\right.
$$

and $N(\lambda)=M(\lambda) G(\lambda)$, where $K=-X^{-1} E_{b}^{-*} C_{b}^{*} J$, and $W$ is any $J$ unitary matrix satisfying $W^{*} J W=$ $J$. If $G$ has real coefficients then the class of solutions with real coefficients is also given by the above formulas where now all the coefficients can be chosen real.

If in the statement of the above theorem we add the condition $X>0$ or $X<0$ we obtain the solution to the LCF with $J$ lossless or $J$ expansive denominator, respectively (see [4]). Notice that for the chosen partition (10) the equation (12) has always a unique solution, and thus the above theorem is an effective tool for checking the existence of and computing the solutions to the LCF with $J$ all-pass denominator or, as a particular case, with $J$ lossless denominator.

\section{LCF with $J$ all-pass denominators with respect to the unit circle}

In this section we give the discrete-time version of the results presented in Section 6. More precisely, we solve the least order LCF problem with the additional requirement on the denominator to have another type of symmetry, namely to be $J$ all-pass with respect to the unit circle. Throughout this section we take the disjoint partition $\overline{\mathbb{C}}=\Gamma_{g} \cup \Gamma_{b}$ defined by

$$
\Gamma_{b}:=\mathbb{D}^{c}\left(\text { or } \Gamma_{b}:=\mathbb{D}\right), \quad \Gamma_{g}:=\overline{\mathbb{C}} \backslash \Gamma_{b} .
$$

Similarly as for the symmetry discussed in the previous section, it is not always possible to solve the LCF over $\Gamma_{g}$ with least order $n_{b}$. Again, when this is possible, we call the factorization canonical, otherwise we call it noncanonical.

Since our theory encompasses the cases in which $M(z)$ has to cancel a pole of $G$ at $\infty$ or at 0 , it follows that $M(z)$ or $M^{-1}(z)$ could, in general, be improper. Therefore, to achieve the full generality we have to use for $M(z)$ a descriptor representation of type (4), with singular $E$. The following result gives a characterization of $J$ all-pass rational matrices (possible improper) without poles at 1 in terms of associated realizations. 
Lemma 6.1 Let $M(z)$ be a square invertible rational matrix, without poles at 1 , having a minimal realization

$$
M(z):=\left[\begin{array}{c|c}
A-z E & (1-z) B \\
\hline C & D
\end{array}\right] .
$$

Then $M$ is $J$ all-pass with respect to the unit circle if and only if $D^{*} J D=J$ and there exists an invertible Hermitic matrix $X$ such that $E^{*} X E-A^{*} X A-C^{*} J C=$ 0 , and $C-D J B^{*} X(E-A)=0$.

The following theorem gives a complete solution to the LCF with $J$ all-pass denominator of McMillan degree $n_{b}$ (the canonical case).

Theorem 6.2 Given an arbitrary rational matrix $G(z)$ and a disjoint partition $\overline{\mathbb{C}}=\Gamma_{g} \cup \Gamma_{b}$ defined by (14), let $n_{b}$ be the number of poles of $G$ in $\Gamma_{b}$. Assume (5) is a minimal descriptor realization of $G$ separated with respect to the given partition and satisfying the condition (6). Then the LCF with $J$ all-pass denominator with respect to the unit circle has a solution $G(z)=M^{-1}(z) N(z)$ of least order $n_{b}$ if and only if the Stein equation

$$
E_{b}^{*} X E_{b}-A_{b}^{*} X A_{b}-C_{b}^{*} J C_{b}=0
$$

has an invertible Hermitic solution $X$. In this case the class of all solutions is given by

$$
M(z)=\left[\begin{array}{c|c}
A_{b}-z E_{b}+K C_{b}(1-z) & K(1-z) \\
\hline W C_{b} & W
\end{array}\right]
$$

and $N(\lambda)=M(\lambda) G(\lambda)$, where $K=-X^{-1}\left(E_{b}-\right.$ $\left.A_{b}\right)^{-*} C_{b}^{*} J$ and $W$ is any $J$ unitary matrix satisfying $W^{*} J W=J$. If $G$ has real coefficients then the class of solutions with real coefficients is also given by the formulas above where now all the coefficients can be chosen real.

If in the statement of the above theorem we add the condition $X>0$ or $X<0$ we obtain the solution to the LCF with $J$ lossless or $J$ expansive denominator, respectively. Notice that for the chosen partition (14) the equation (16) has always a unique solution, and thus the above theorem is an effective tool for checking the existence of and for computing solutions to the LCF with $J$ all-pass denominator or, as a particular case, with $J$ lossless denominator.

\section{Numerical examples}

Example 1. least order factorization with proper factors. Consider the improper rational matrix $G(\lambda)=$ $\left[\begin{array}{cc}\lambda^{2} & \frac{\lambda}{\lambda-1} \\ 0 & \frac{1}{\lambda}\end{array}\right]$ having finite poles at $\lambda_{1}=1$ and $\lambda_{2}=0$ and two poles at infinity, and let $\Gamma_{g}=\mathbb{C}^{-}$. A minimal order descriptor realization for $G$ in the form (5) is given by

$$
\begin{aligned}
& {\left[\begin{array}{c|c}
A_{b}-\lambda E_{b} & A_{b g}-\lambda E_{b g} \\
\hline O & A_{g}-\lambda E_{g}
\end{array}\right]=\left[\begin{array}{cccc|c}
-\lambda & 1 & 0 & 0 & 0 \\
0 & 1-\lambda & 0 & 0 & 0 \\
0 & 0 & 1 & -\lambda & 0 \\
0 & 0 & 0 & 1 & -\lambda \\
\hline 0 & 0 & 0 & 0 & 1
\end{array}\right]}
\end{aligned}
$$

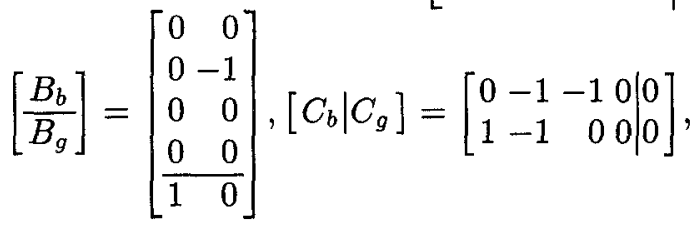

$D=\left[\begin{array}{ll}0 & 1 \\ 0 & 0\end{array}\right]$. To compute a least order LCF over $\Gamma_{g}$ we use Theorem 4.2 and determine first a matrix $K$ such that $\Lambda\left(A_{b}-\lambda E_{b}+K C_{b}(\alpha-\beta \lambda)\right)=$ $\{-1,-1,-1,-1\}$, where we chose for convenience $\alpha=$ -2 and $\beta=1$. By using the Lemma 4.1 for the dual pair $\left(A_{b}^{T}-\lambda E_{b}^{T}, C_{b}^{T}\right)$ we can assign the eigenvalues for the modified pair $\left(A_{z}-\lambda E_{z}, C_{z}\right):=\left(\alpha A_{b}^{T}-\beta E_{b}^{T}-\right.$ $\left.\lambda\left(\beta A_{b}^{T}-\alpha E_{b}^{T}\right),\left(\alpha^{2}+\beta^{2}\right) C_{b}^{T}\right)$ to $\Gamma_{z}:=\{3,3,3,3\}$, where $E_{z}$ is nonsingular. By using a pole assignment algorithm for generalized eigenvalues we have determined $K=\left[\begin{array}{cccc}-8 & -8 & 6 & 3 \\ 1 & 0 & 0 & 0\end{array}\right]^{T}$. Finally, we get the following solution to the least order LCF over $\Gamma_{g}$ :

$N=\left[\begin{array}{cc}\frac{(\lambda-1) \lambda^{2}}{3(\lambda+1)^{3}} & \frac{\lambda}{3(\lambda+1)^{3}} \\ 0 & \frac{1}{2(\lambda+1)}\end{array}\right], M=\left[\begin{array}{cc}\frac{\lambda-1}{3(\lambda+1)^{3}} & 0 \\ 0 & \frac{\lambda}{2(\lambda+1)}\end{array}\right]$

where the least order of the LCF is clearly 4 .

Example 2. least order coprime factorization with $J$ all-pass denominator with respect to the unit circle

Consider the TFM of a discrete-time system $G(z)=\left[\begin{array}{cc}z^{2} & \frac{1}{z-2} \\ 0 & z\end{array}\right]$ having an unstable finite pole at $z_{1}=2$ and three poles at infinity, and let $\Gamma_{b}:=\mathbb{D}^{c}$. A minimal order descriptor realization for $G$ in the form (5) is given by $\left[\begin{array}{c|c}A_{b}-z E_{b} & A_{b g}-z E_{b g} \\ \hline O & A_{g}-z E_{g}\end{array}\right]=\left[\begin{array}{rrrr|rr}2-z & 0 & 0 & 0 & 0 \\ 0 & 1 & -z & 0 & 0 & 0 \\ 0 & 0 & 1 & 0 & -z & 0 \\ 0 & 0 & 0 & 1 & 0 & -z \\ \hline 0 & 0 & 0 & 0 & 1 & 0 \\ 0 & 0 & 0 & 0 & 0 & 1\end{array}\right]$

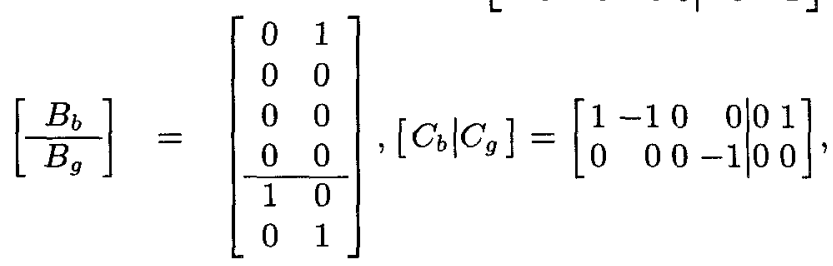


$D=\left[\begin{array}{ll}0 & 1 \\ 0 & 0\end{array}\right]$. We get for the solution of $(16)$ and the corresponding $K$ in Theorem 6.2

$$
X=\left[\begin{array}{cccc}
-1 / 3 & 1 / 2 & 1 / 4 & 0 \\
1 / 2 & -1 & 0 & 0 \\
1 / 4 & 0 & -1 & 0 \\
0 & 0 & 0 & 1
\end{array}\right], K=\left[\begin{array}{rr}
-12 & 0 \\
-5 & 0 \\
-2 & 0 \\
0 & 1
\end{array}\right]
$$

respectively. The factors $N(z)$ and $M(z)$ resulting from Theorem 6.2 are

$$
N=\left[\begin{array}{cc}
\frac{2-z}{2 z-1} & \frac{1}{z^{2}(1-2 z)} \\
0 & 1
\end{array}\right], M=\left[\begin{array}{cc}
\frac{2-z}{z^{2}(2 z-1)} & 0 \\
0 & \frac{1}{z}
\end{array}\right]
$$

\section{Conclusions}

We have presented a comprehensive theory of minimal degree coprime factorization of rational matrices over a given domain of the closed complex plane. The given formulas allow to compute the coprime factors either by an one-shot approach, or recursively by dislocating one pole (or a pair of poles) at a time (see for example the pole dislocation technique in [9]). In general, the one-shot approach brings advantages in terms of numerical reliability, efficiency and versatility as any algorithm for pole assignment may be used, while the recursive method is more advantageous if a certain form of the coprime factors is needed at futher compuatational steps. Moreover, the one-shot approach is applicable for a much larger class of problems than the recursive methods. The recursive techniques are applicable only when the involved Lyapunov equations have sign definite solutions (positive or negative), and then these equations are solved only implicitly. However, the recursive techniques can not be employed in the more general cases discussed in this paper, not even in the canonical $J$ all-pass case. Essentially, the recursive techniques fail since a $J$ all-pass rational matrix can not be written in general as a product of elementary $J$ all-pass factors. In this respect this paper elucidates in which cases the recursive algorithms proposed in [11], [10] are applicable and provide the right answer.

A particular feature of the results developed in this paper is that the methods are exactly tailored to the dimension of the problem to be solved avoiding unnecessary redundancy. As an example, the solution of a full order Riccati equation (order equal to the McMillan degree of the rational matrix to be factored) that is usually employed in such factorizations (see for example [4], [16]) is completely avoided. Instead, we solve a Lyapunov equation of lower dimension with the benefits of increased numerical accuracy and computational efficiency.
The theory presented here has been already applied as a preliminary step to the computation of the most general inner-outer, spectral [5], and $J$ lossless factorizations and it is a promising step towards computing the more general $J$ spectral factorizations, either canonical or noncanonical. What concerns the noncanonical case, all the details have been carried out and will be reported in a forthcoming paper.

\section{References}

[1] J.A. Ball, J. Kim, L. Rodman, M. Verma, Minimal degree coprime factorization of rational matrix functions, Lin. Alg. \& Appl., 186:117-164, 1993.

[2] H. Bart, I. Gohberg, M.A. Kaashoek. Constructive Methods of Wiener-Hopf Factorization. Birkhäuser, Basel, OT 21, 1986.

[3] I. Gohberg, M.A. Kaashoek, A.C.M. Ran. Partial pole and zero displacement by cascade connection. SIAM J. Matrix Anal. Appl., 10(3):316-325, 1989.

[4] H. Kimura. Chain-Scattering Approach to $H_{\infty^{-}}$ Control. Birkhäuser, Boston, 1996.

[5] C. Oară and A. Varga. Inner-outer factorization of rational matrices: the general case. In Proceedings of the MTNS'98, Padova, 1998.

[6] C. Oară Minimal Factorization of Rational Matrices: The General Case. In Proceedings of the MTNS'98, Padova, 1998.

[7] H. H. Rosenbrock. State-Space and Multivariable Theory. Wiley, New York, 1970.

[8] P. Van Dooren. The generalized eigenstructure problem in linear systems theory. IEEE Trans. Autom. Control, 26:111-129, 1981.

[9] P. Van Dooren. Rational and polynomial matrix factorizations via recursive pole-zero cancellation. $\mathrm{Lin}$. Alg. \& Appl., 137/138:663-697, 1990.

[10] A. Varga. Computation of coprime factorizations of rational matrices. LAA, 271:83-115, 1998.

[11] A. Varga and T. Katayama. Computation of Jinner-outer factorizations of rational matrices. Int. $J$. Robust and Nonlinear Control, 1997. (to appear).

[12] G. Verghese, B. Lévy, and T. Kailath. A generalized state-space for singular systems. IEEE Trans. Autom. Control, AC-26:811-831, 1981.

[13] G. Verghese, P. Van Dooren, and T. Kailath. Properties of the system matrix of a generalized statespace system. Int. J. Control, 30:235-243, 1979.

[14] M. Vidyasagar. Control System Synthesis: A Factorization Approach. MIT Press, Cambridge, 1985. [15] X. Xin and H. Kimura. $\left(J, J^{\prime}\right)$ lossless factorization for descriptor systems. Lin. Alg. \& Appl., 205206:1289-1318, 1994.

[16] X. Xin and H. Kimura. Singular $\left(J, J^{\prime}\right)$ lossless factorization for strictly proper functions. Int. J. Control, 59:1383-1400, 1994. 